\title{
Correspondence
}

\section{Breaking a species barrier by enabling hybrid recombination}

G. Ozan Bozdag ${ }^{\dagger, 1,2}$, Jasmine Ono ${ }^{\dagger, 3,9,{ }^{*}}$, Jai A. Denton ${ }^{4}$, Emre Karakoc ${ }^{5}$, Neil Hunter ${ }^{6,7}$, Jun-Yi $\mathrm{Leu}^{8}$, and Duncan Greig ${ }^{2,3}$

${ }^{1}$ School of Biological Sciences, Georgia Institute of Technology, Atlanta, GA, 30332, USA

${ }^{2}$ Experimental Evolution Research Group, Max Planck Institute for Evolutionary Biology, Plön, 24306, Germany

${ }^{3}$ Centre for Life's Origins and Evolution, Department of Genetics, Evolution and Environment, University College London, London, WC1E 6BT, United Kingdom

${ }^{4}$ Genomics \& Regulatory Systems Unit, Okinawa Institute of Science \& Technology, Onna-son, 904-0495, Japan

${ }^{5}$ Wellcome Sanger Institute, Wellcome Genome Campus, Hinxton, Cambridge, CB10 1SA, United Kingdom

${ }^{6}$ Howard Hughes Medical Institute, University of California, Davis, Davis, CA, 95616, USA

${ }^{7}$ Department of Microbiology \& Molecular Genetics, University of California, Davis, Davis, CA, 95616, USA

${ }^{8}$ Institute of Molecular Biology, Academia Sinica, Taipei, 11529, Taiwan

${ }^{9}$ Lead Contact

†These authors contributed equally to this work.

*Correspondence: j.ono@,ucl.ac.uk

Keywords: yeast, speciation, anti-recombination, reproductive isolation, hybrid fertility, hybrid recombination, Saccharomyces

eTOC

Bozdag et al. show that relieving anti-recombination during hybrid meiosis dissolves the reproductive barrier between two yeast species by enabling their chromosomes to recombine and segregate properly. This confirms anti-recombination as the major cause of hybrid sterility and allows interbreeding of distant species for research or commercial use. 


\section{MAIN TEXT}

Hybrid sterility maintains reproductive isolation between species by preventing them from exchanging genetic material ${ }^{1}$. Anti-recombination can contribute to hybrid sterility when different species' chromosome sequences are too diverged to cross-over efficiently during hybrid meiosis, resulting in chromosome mis-segregation and aneuploidy. The genome sequences of the yeasts Saccharomyces cerevisiae and Saccharomyces paradoxus have diverged by about $12 \%$ and their hybrids are sexually sterile: nearly all of their gametes are aneuploid and inviable. Previous methods to increase hybrid yeast fertility have targeted the anti-recombination machinery, enhancing meiotic crossing over but also having counteracting detrimental effects on gamete viability due to increased mutagenesis ${ }^{2}$ and ectopic recombination ${ }^{3}$. Therefore the role of anti-recombination has not been fully revealed, and it is often dismissed as a minor player in speciation $^{1}$. By repressing two genes, SGS1 and MSH2, specifically during meiosis whilst maintaining their mitotic expression, we were able to increase hybrid fertility 70 -fold, to the level of non-hybrid crosses, confirming that anti-recombination is the principal cause of hybrid sterility. Breaking this species barrier allows us to generate, for the first time, viable euploid gametes containing recombinant hybrid genomes from these two highly diverged parent species.

Species are formed and maintained by the restriction of gene flow between diverging populations. Barriers to gene flow can be physical, such as geographic distance, or they can be properties of the species themselves. Here, we focus on one such barrier to gene flow, hybrid sterility. Hybrid sterility can be caused by a variety of mechanisms that can generally be classified into incompatibilities between diverged chromosomes (such as large-scale chromosomal rearrangements and anti-recombination) and incompatibilities between individual genes from the diverging populations ${ }^{1}$. 
Here we show that repressing anti-recombination dissolves the reproductive barrier between two yeast species, S. cerevisiae and S. paradoxus, increasing their production of viable hybrid gametes by 70 -fold (Figure 1A). We did this by repressing the meiotic expression of just two highly conserved genes, $S G S 1$ and $M S H 2$. Msh2 is a component of the mismatch repair system that removes base-pair mismatches in duplex DNA, both to repair misincorporations in newly synthesized DNA and to inhibit recombination between diverged sequences (antirecombination). The former activity reduces mutations, and the latter can help maintain genome integrity by limiting ectopic recombination between non-homologous chromosomes and dispersed repeats ${ }^{3}$. Sgs1 is a DNA helicase that is assumed to act downstream of mismatch recognition by Msh2 to unwind nascent recombination intermediates containing a high density of mismatches ${ }^{4}$, but also plays a more general function in recombination to disassemble jointmolecule intermediates that could lead to crossovers 5 . Thus, although completely deleting $\mathrm{MSH} 2$ enhances meiotic recombination between the diverged chromosomes of $S$. cerevisiae x $S$. paradoxus hybrids, increasing proper chromosome segregation and therefore hybrid spore viability ${ }^{2}$, this benefit is countered by elevated mutagenesis and genome instability in mitotically dividing cells, which reduces viability. We therefore replaced the native promoters of $M S H 2$ and $S G S 1$ with the $C L B 2$ promoter, which represses gene expression during meiosis but not mitosis ${ }^{6}$. Meiotic repression of either gene alone significantly increased hybrid spore viability (Figure 1A, MSH2 $p=7.99 \times 10^{-6} ; S G S 1 p<2.2 \times 10^{-16}$ ). Overall spore viability rose from $0.46 \%$ in the wild-type hybrid to $3.18 \%$ in the $p C L B 2-M S H 2$ strain and to $20.08 \%$ in the $p C L B 2-S G S 1$ strain. Spore viability was further improved to $32.65 \%$ when both genes were repressed $(p<$ $\left.2.2 \times 10^{-16}\right)$. Although hybrid fertility was not increased to the level of the parents- the $S$. cerevisiae and S. paradoxus parent fertilities were $83.75 \%$ and $92.25 \%$, respectively—it was 
well within the range of fertilities of non-hybrid crosses formed from diverged populations of one species or the other, e.g. 32-87\% for S. paradoxus or S. cerevisiae crosses with collinear genomes $^{7}, 14-86 \%$ for wild $S$. paradoxus crosses $^{8,9}$. These results show that anti-recombination determines most of the hybrid sterility barrier between our $S$. cerevisiae and $S$. paradoxus strains.

This remarkable restoration of hybrid fertility allowed us to produce a large sample of perfectly euploid hybrid gametes. Any viable gametes produced by a hybrid are usually aneuploid ${ }^{2}$, and this remains the case even when $M S H 2$ is knocked out ${ }^{10}$. By dramatically improving hybrid fertility, we significantly increased the production of hybrid tetrads in which all four spores were viable from $0 \%$ to $5.3 \%$ (Fig. 1B, 0/269 versus 108/2037 respectively, $p=$ $\left.2.04 \times 10^{-4}\right)$. Because all chromosomes are essential in yeast, we can infer that these full tetrads contain only euploid hybrid gametes. Generation of these hybrids enables the unambiguous analysis of recombination and trait mapping, both of which were previously confounded by aneuploidy in sampled hybrid spores ${ }^{10}$.

Finally, in order to map the genome-wide distribution of crossovers in our $p C L B 2-M S H 2$ pCLB2-SGS1 double mutant hybrid, we sequenced the genomes of the 336 hybrid spores from 84 fully viable tetrads. We found that crossing over was much increased compared to wild-type hybrid strains with an average of 18.9 crossovers per spore. Figure S1A shows that recombination in our manipulated hybrid followed the normal, non-hybrid pattern of a higher crossover density $(\mathrm{cM} / \mathrm{kbp})$ on smaller chromosomes than larger chromosomes $(S$. cerevisiae slope $=-0.00013, p=0.0032$; hybrid mutant slope $=-4.22 \times 10-5, p=0.048)$, in contrast to the wild type, unmanipulated hybrid measured by Kao et al. $(2010)^{10}\left(\right.$ slope $\left.=-3.82 \times 10^{-8}, p=0.99\right)$. Sequencing the genomes of our recombinant hybrid spores revealed that the suppression of anti- 
recombination activity was evenly distributed across the genomes of both species, rather than being locally enriched at hotspot regions (Figure S1A).

This study shows that repressing the meiotic expression of just two genes, SGS1 and MSH2, overcomes the anti-recombination barrier between two yeast species, restoring the fertility of their hybrids to intraspecific levels, and allowing them to produce viable, euploid, recombinant gametes (Figure 1C, Figure S1B). We demonstrate directly that anti-recombination is the major cause of post-zygotic reproductive isolation between these species. By enabling recombination between such diverged species, our method can be used to identify any intrinsic genetic incompatibilities or speciation genes, to map the genetics underlying diverged phenotypes, or to produce recombinant hybrids with novel properties for commercial or research use.

\section{FIGURE LEGENDS}

Figure 1: Restoration of hybrid fertility by meiotic repression of MSH2 and SGS1. A. Percentages are spore viabilities of the indicated hybrid strains. Both single mutants and the double mutant have significant increases in spore viability. Numbers in parentheses indicate the total number of dissected spores checked for viability. B. Meiotic repression of SGS1 and MSH2 increases the frequency of 4-spore tetrads. Full data, including other strains, can be found in the associated Dryad package. C. An example recombination map of a single tetrad. Gametes were genotyped by open reading frame (ORF) into one of the two species, ensuring a 2:2 segregation of species identity at each ORF. Only ORFs that were shared and co-linear between the two species were considered. Segments are coloured according to their parent of origin.

\section{SUPPLEMENTAL INFORMATION}

Supplemental Information includes one figure, experimental procedures, primer sequences, strain details, resource availability, acknowledgements, author contributions, and supplemental references and can be found with this article online. 


\section{DECLARATION OF INTERESTS}

The authors declare no competing interests.

\section{REFERENCES}

1 Coyne, J.A., and Orr, H.A. (2004). Speciation (Sinauer Associates).

2 Hunter, N., Chambers, S.R., Louis, E.J., and Borts, R.H. (1996). The mismatch repair system contributes to meiotic sterility in an interspecific yeast hybrid. EMBO J. 15, 1726-1733.

3 Myung, K., Datta, A., Chen, C., and Kolodner, R.D. (2001). SGS1, the Saccharomyces cerevisiae homologue of BLM and WRN, suppresses genome instability and homeologous recombination. Nat. Genet. 27, 113-116.

4 Chakraborty, U., and Alani, E. (2016). Understanding how mismatch repair proteins participate in the repair/anti-recombination decision. FEMS Yeast Res. 16, fow071.

5 Zakharyevich, K., Tang, S., Ma, Y., and Hunter, N. (2012). Delineation of joint molecule resolution pathways in meiosis identifies a crossover-specific resolvase. Cell 149, 334-347.

6 Grandin, N., and Reed, S.I. (1993). Differential function and expression of Saccharomyces cerevisiae B-type cyclins in mitosis and meiosis. Mol. Cell. Biol. 13, 2113-2125.

7 Liti, G., Barton, D.B.H., and Louis, E.J. (2006). Sequence diversity, reproductive isolation and species concepts in Saccharomyces. Genetics 174, 839-850.

8 Greig, D., Travisano, M., Louis, E.J., and Borts, R.H. (2013). A role for the mismatch repair system during incipient speciation in Saccharomyces. J. Evol. Biol. 16, 429-443.

9 Charron, G., Leducq, J.B., and Landry, C.R. (2014). Chromosomal variation segregates within incipient species and correlates with reproductive isolation. Mol. Ecol. 23, 4362-4372. 
10 Kao, K.C., Schwartz, K., and Sherlock, G. (2010). A genome-wide analysis reveals no nuclear Dobzhansky-Muller pairs of determinants of speciation between $S$. cerevisiae and $S$. paradoxus, but suggests more complex incompatibilities. PLoS Genet. 6, e1001038. 


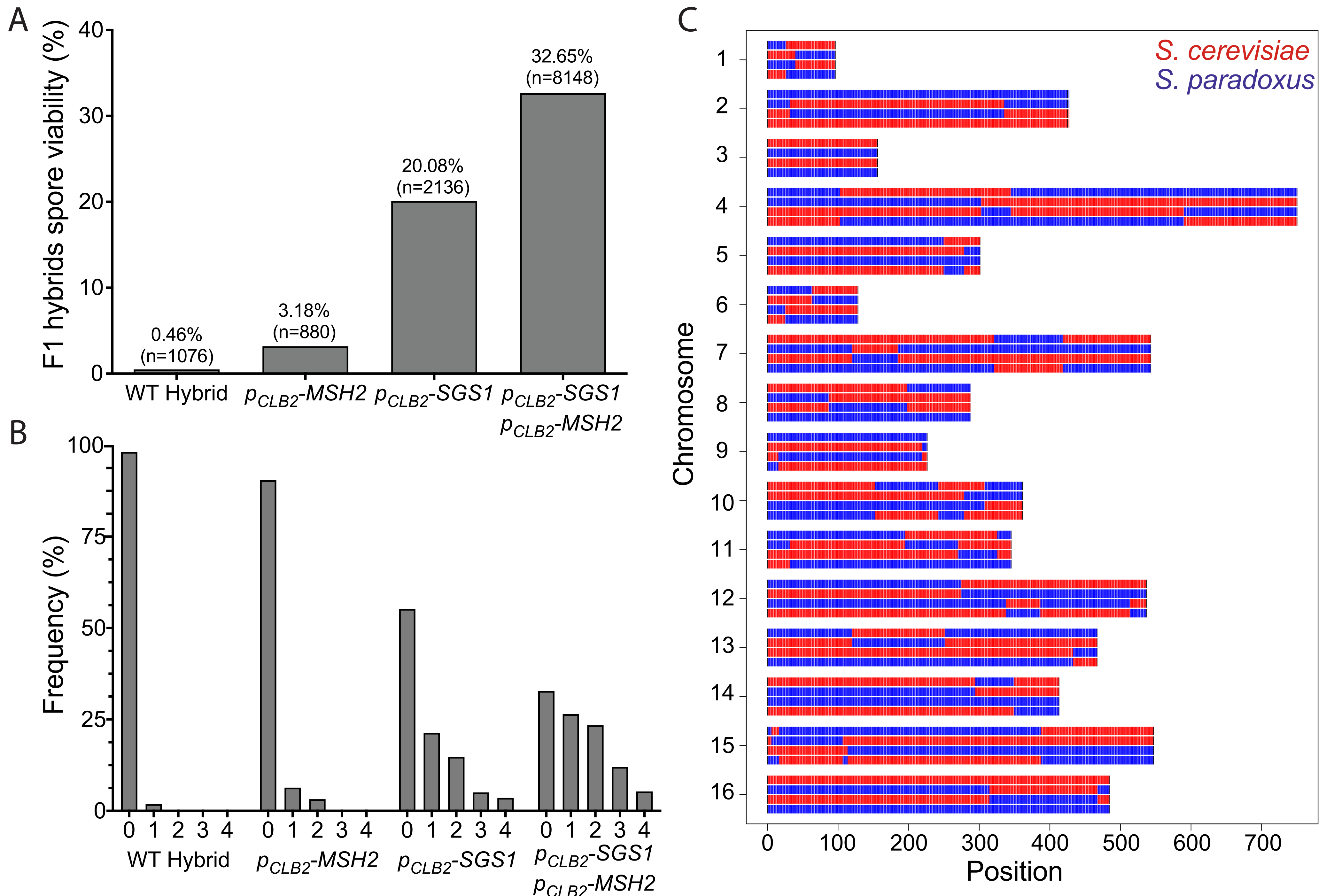


SUPPLEMENTAL INFORMATION: Breaking a species barrier by enabling hybrid recombination

G. Ozan Bozdag, Jasmine Ono, Jai A. Denton, Emre Karakoc, Neil Hunter, Jun-Yi Leu, and Duncan Greig
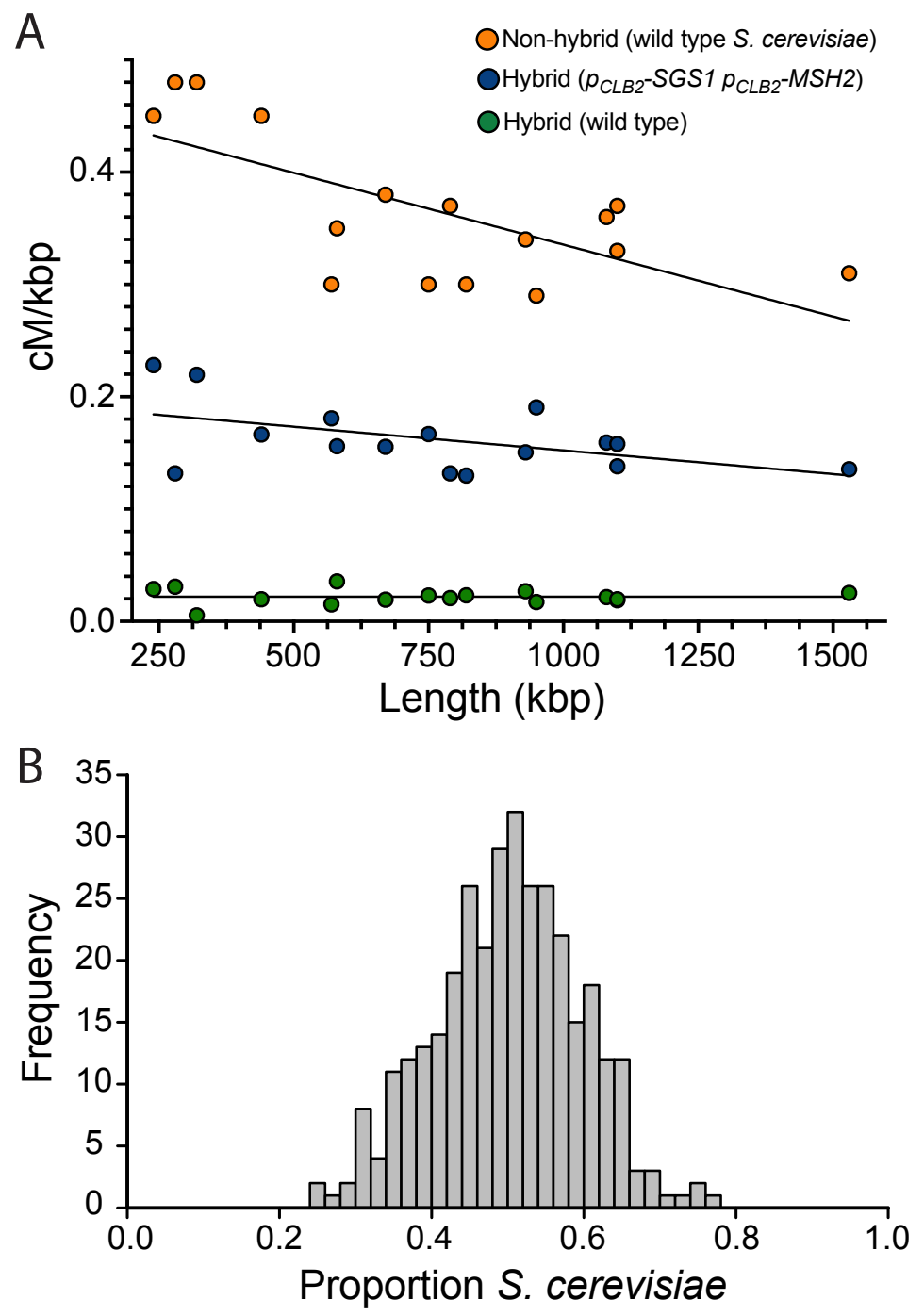

Figure S1: Mutant hybrid yeast show similar patterns of recombination to non-hybrid wild type. A. Hybrid double mutants (blue) have overall lower recombination than observed in $S$. cerevisiae (yellow), but much higher recombination than wild type hybrids (green). Similar patterns of more recombination per kbp in shorter chromosomes is observed for both $S$. cerevisiae and the hybrid mutant. Approximate chromosome lengths and genetic map distances were obtained for S288C (SGD) ${ }^{\mathrm{S} 14}$ and used for all strains even though hybrids are expected to have a mix of chromosome lengths. The hybrid wild type data is from Kao et al. $2010^{\mathrm{S} 15}$. B. The recombinant spores produced by our manipulated hybrid were normally distributed for inheritance of each species' genome (variance in proportion of the genome inherited from $S$. cerevisiae $=0.0090)$. 


\section{SUPPLEMENTAL EXPERIMENTAL PROCEDURES}

\section{Strains}

We used as a template a previously constructed Saccharomyces cerevisiae strain NHY 2039, in which the promoter of $S G S 1$ had been replaced by the $C L B 2$ promoter ${ }^{S 1, S 2}$ using the pFA6aKANMX4pCLB2-3HA construct created by Lee and Amon $(2003)^{\mathrm{S} 3}$. We amplified the $S$. cerevisiae CLB2 promoter and the KANMX4 drug resistance marker out of NHY2039 (i.e. YDG832) using primer pairs (see below) that allowed us to transform it in place of the natural promoters of $M S H 2$ and SGS1 in both S. cerevisiae (W303 background) and S. paradoxus (N17 background).

The resulting S. cerevisiae and S. paradoxus haploid strains YDG968 and YDG969 were crossed together producing an F1 hybrid diploid YDG982 in which both homologous copies of both $S G S 1$ and $M S H 2$ were under the control of the CLB2 promoter, repressing the expression of these genes during meiosis (see Extended Data Figure 1 in the Dryad package and strains listed below for details). To obtain a non-hybrid, double-mutant (i.e. $\left.P_{C L B 2}-M S H 2, P_{C L B 2}-S G S 1\right)$ control strain under the S. paradoxus background, we crossed haploid strains YDG967 and YDG968. Next, we crossed YDG969 and YDG970 strains to obtain a similar non-hybrid, double mutant (i.e. $\left.P_{C L B 2}-M S H 2, P_{C L B 2}-S G S 1\right)$ control strain for the $S$. cerevisiae background. Finally, to obtain a wild-type hybrid control strain (i.e. without CLB2 promoter replacement), we crossed haploid strains YDG391 (S. paradoxus) and YDG542 (S. cerevisiae), and selected for diploid clones (to form YDG853).

\section{Fertility}

We induced meiosis and sporulation by incubating the hybrid diploid (YDG982) in $3 \mathrm{ml} \mathrm{KAc}$ ( $2 \%$ potassium acetate sporulation media) for four days at room temperature with vigorous shaking. To digest the ascus walls of the hybrid ascospores, we incubated them in 1 unit (per 10 
l) zymolyase (Zymo Research EU, Freiburg, Germany) for 30 minutes. After enzymatic digestion of the ascus walls, we placed the four spores of each tetrad onto YEPD ( $2 \%$ glucose, $1 \%$ yeast extract, $2 \%$ peptone, $2 \%$ agar) plates using an MSM400 tetrad dissection microscope (Singer Instruments, Watchet, UK). Plates containing dissected tetrads were incubated at $30{ }^{\circ} \mathrm{C}$ before examining them for visible colonies founded by germinating spores.

We defined fertility as the proportion of viable gametes, i.e. the number of spores that germinated and formed colonies visible to the naked eye after two days, divided by the total number of spores that were dissected. For the hybrid crosses, we dissected a large number of spores ( $\geqq 880$, see Extended Data Tables 1 and 2 in the Dryad package). This was necessary for the hybrid crosses because they were known to have low gamete viability. For the non-hybrid crosses, we only dissected 360-400 spores. Because the non-hybrid crosses had much higher rates of gamete viability than the hybrid crosses, dissecting a lower number of spores was sufficient to obtain a good estimate of their true fertility (all standard error $<3 \%$ ). Only technical replicates (repeated meioses of the same original diploid strain) were performed and they were all considered to be part of a single sample.

\section{Sequencing and genotyping}

To ensure that the hybrid gametes we sequenced were euploid, we only genotyped gametes from tetrads that contained four viable spores. In order to maximize useable data from a single lane of sequencing, we limited our sample size to 94 tetrads. Again, repeated meiosis of a single diploid strain were performed and were all considered to be part of a single sample. We extracted DNA from all 376 colonies from 94 tetrads (in addition to two non-hybrid control tetrads) using MasterPure ${ }^{\mathrm{TM}}$ Yeast DNA Purification Kit (Epicentre, Biozyme Biotech, Oldendorf, Germany). To prepare the samples for sequencing, we used double digestion based RAD-tag library preparation method ${ }^{\mathrm{S} 4, \mathrm{~S} 5, \mathrm{~S} 6}$. We digested $50 \mathrm{ng}$ of DNA from each colony using restriction 
enzymes Csp6I and PstI and ligated adapters (adapterX_TagY_fq and adapterX_TagY_rv) in the same reaction at $37^{\circ} \mathrm{C}$ for two hours. We cleaned up the excess adapters, enzymes, and fragments smaller than 300 bp by using Ampure beads at a 1:1 ratio. Next we mixed Phusion Hot Start II High-Fidelity DNA Polymerase $(2 \mathrm{U} / \mu \mathrm{l})$, adding P5 and P7 primers at $10 \mathrm{mM}$ concentration, dNTPs (2mM per dNTP), and 5X Phusion HF Buffer to amplify the target regions $^{\mathrm{S} 4, \mathrm{~S} 7} .30 \mu \mathrm{PCR}$ mixtures were amplified using an initial $98^{\circ} \mathrm{C}$ incubation for $30 \mathrm{~s}$, followed by 25 cycles of $98^{\circ} \mathrm{C}$ for $10 \mathrm{~s}, 68^{\circ} \mathrm{C}$ for $15 \mathrm{~s}, 72^{\circ} \mathrm{C}$ for $30 \mathrm{~s}$, and then a final extension at $72^{\circ} \mathrm{C}$ for 5 mins. To sequence the tagged samples, we mixed all tagged samples in one pool. All samples were multiplexed using combinations of 24 unique barcodes, therefore reads from a single sequencing reaction had unique reverse and forward tags, which allowed us to distinguish the samples. We used the MiSeq platform to obtain 300 bp paired-end reads.

To map the reads, we assembled two simplified co-linear reference genomes consisting of the coding DNA only from the set of open reading frames (ORFs) shared between $S$. cerevisiae and $S$. paradoxus, removing ORFs that were present in one species but not in another or which were not co-linear (based on SGRP sequencing added 10/10/08 ${ }^{\mathrm{S} 8, \mathrm{~S} 9}$ ). We mapped reads to these reference genomes using bwa-mem ${ }^{\mathrm{S} 10}$. At this point, we excluded 10 tetrads due to poor sequencing coverage and quality, leaving us with 336 samples from 84 tetrads. We assigned ORFs to one species or the other using two simplifying assumptions: that no non-Mendelian segregation occurred and that recombination occurred only in intergenic regions. Thus, if all four spores of a tetrad contained reads mapping to a given ORF of one or both species, the two spores with the highest proportion of reads mapping to one species' ORF would have it assigned to that species and the other two would have the ORF assigned to the other species. If the four spores within a tetrad did not all contain reads for an ORF, it would be assigned to the same species as the neighboring ORF. These genotyping rules produced a recombination map (see Figure 1C for 
an example, full data available from Dryad) of the four spores within each tetrad at ORF-level resolution, with no gain or loss of genetic material (i.e. no gene-conversion). To visualize the genotype of these hybrid spores, we used PlotTetradSeq in the Recombine package in $\mathrm{R}^{\mathrm{S} 11}$.

\section{Statistics}

$\mathrm{X}^{2}$ contingency tests for viability statistics and comparing the production of 4-spore tetrads between strains were performed using Pearson's $\mathrm{X}^{2}$ test with Yates' continuity correction using the function 'chisq.test' from the package stats in $\mathrm{R}^{\mathrm{S} 12}$. The standard error for the viability data was calculated manually in $\mathrm{R}$ using the formula $\mathrm{SE}=\operatorname{squareroot}(\mathrm{p}(1-\mathrm{p}) / \mathrm{n})$. The $95 \%$ confidence interval was calculated using the Agresti-Coull method in the function 'binom.confint' from the package binom in $\mathrm{R}^{\mathrm{S} 12}$. Linear regressions looking at each strain's cM/kbp were performed using the 'linregress' function from the scipy.stats package in Python ${ }^{\mathrm{S} 13}$, which fits a linear regression line that minimizes the sum-of-squares for two sets of measurements.

\section{Primers used in this study}

To replace the native promoter of SGS1 with $K A N M X-p C L B 2 S G S 1$ cassette (can be amplified from YDG832 or YDG833):

S. cerevisiae

\section{FkMXpClb2Sgs1Sc}

ATTATTGTTGTATATATTTAAAAAATCATACACGTACACACAAGGCGGTAGAATTCG AGCTCGTTTAAAC

RkMXpClb2Sgs1Sc

TTTAACCATTTGTGCTCCCTTCTTAAGTTATGTGACGGCTTCGTCACCATGCACTGAG CAGCGTAATCT

S. paradoxus

\section{FkMXpClb2Sgs1Sp}

AGTTCAGTGTATATATTTAAAGTCACACGCATACACGCGAATTCGAGCTCGTTTAAA $\mathrm{C}$

or

\section{SpKMXpCLB2sgs1F} CAGCCAGTTTGGAGTCATCAGCTACAGGAAGGGAAAACGAATTCGAGCTCGTTTAA $\mathrm{AC}$ 


\section{RkMXpClb2Sgs1Sp}

TTCAACCATTTGTGCTCCCTTCTTAAGTTATGTGAGGGCTTCGTCACCATGCACTGAG CAGCGTAATCT

To diagnose replacement of native $S G S 1$ promoter with $K A N M X-p C L B 2$ cassette:

\section{RdKanMX}

GTTCGGATGTGATGTGAGAACTG

\section{RdSgs1orfSe}

TGTGCTTTTGGATAGCCTGG

To replace the native promoter of $M S H 2$ with $K A N M X-p C L B 2$ cassette:

S. cerevisiae

FpCLBmsh2c

TTATCTGCTGACCTAACATCAAAATCCTCAGATTAAAAGTGAATTCGAGCTCGTTTA

AAC

\section{RpCLBmsh2c}

ATACATCAGAGAATTTTAGCTCTGGCCTAGTGGAGGACATGCACTGAGCAGCGTAAT CT

S. paradoxus

FpCLBmsh2p

TTATCTGTTGATCTAACATCAAAATCTTCGGATAACAAGTGAATTCGAGCTCGTTTA AAC

\section{RpCLBmsh2p}

ATACATCAGAGAATTTCAGCTCTGGTCTAGTAGAGGACATGCACTGAGCAGCGTAAT CT

To diagnose replacement of native $M S H 2$ promoter with $K A N M X-p C L B 2$ cassette:

$S$. cerevisiae (used with any forward primer that anneals to KANMX cassette).

Rd-pCLBmsh2c

CATAACTTCAGCAGAGTGGC

S. paradoxus

Rd-pCLBmsh2p

ATAATTTCAGCAGAGTGG

Strains used in this study

YDG227: ho MATa lys2 cyh2r (N17)

YDG244: ho MATalpha ura3 cyh2r (N17)

YDG253: ho MATa ura lys his3 leu2 trp ade-2can1r (W303) 
YDG350: ho MATalpha ura3-52 his3-11 leu2-3,112 trp142 ade2-1 can1-100(W303, YSC1059)

YDG391: ho::HYGMX MATalpha ura3::KanMX(N17, NCYC 3708)

YDG542: ho::HYGMX MATa ura3::KanMX ade2-1 (W303, NCYC 3583)

YDG832: ho::hisG MATa ura3(ASma-Pst) HIS4::LEU2-(BamHI; +ori) leu2::hisG pCLB23HA-SGS1::kanMX4 (NHY 2039)

YDG833: ho::hisG MATalpha ura3(LSma-Pst) his4-X::LEU2-(NgoMIV; +ori)—URA3 leu2::hisG pCLB2-3HA-SGS1::kanMX4 (NHY 2040)

YDG853: ho::HYGMX/ho::HYGMX MATa/MATalpha ura3::KanMX/ura3::KanMX ade21/ADE2 (N17 x W303, YDG391 x YDG542)

YDG863: ho MATa ura lys his3 leu2 trp ade-2 can1r pCLB2-3HA-SGS1::kanMX4 (W303)

YDG866: ho MATa ura his3 leu2::NAT trp ade-2 can1r pCLB2-3HA-SGS1::kanMX4 (W303)

YDG905: ho MATalpha ura3 cyh2r pCLB2-3HA-SGS1::kanMX4 (N17)

YDG912: ho/ho MATa/MATalpha ura/ura3 his3/HIS3 leu2::NAT/LEU2 trp/TRP ade-2/ADE can1r/CAN1 CYH2/cyh2r pCLB2-3HA-SGS1::kanMX4/pCLB2-3HA-SGS1::kanMX4 (W303 x N17, YDG866 x YDG905)

YDG959: ho MATa lys2 cyh2r pCLB2-3HA-MSH2::kanMX4 (N17)

YDG960: ho MATalpha ura3-52 his3-11 leu2-3,112 trp142 ade2-1 can1-100 pCLB2-3HAMSH2::kanMX4 (W303, YSC1059)

YDG961: ho MATa lys2 cyh2r pCLB2-3HA-SGS1::kanMX4 (N17)

YDG962: ho MATalpha ura3-52 his3-11 leu2-3,112 trp1A2 ade2-1 can1-100 pCLB2-3HASGS1::kanMX4 (W303, YSC1059)

YDG964: ho/ho MATa/MATalpha URA3/ura3-52 lys2/LYS2 HIS3/his3-11 LEU2/leu2-3,112 TRP1/trp1 2 ADE2/ade2-1 CAN1/can1-100 cyh2r/CYH2 pCLB2-3HAMSH2::kanMX4/pCLB2-3HA-MSH2::kanMX4 (N17 x W303, YDG959 x YDG960) 
YDG965: ho/ho MATalpha/MATa ura3/URA3 LYS2/lys2 cyh2r/cyh2r pCLB2-3HA-

SGS1::kanMX4/pCLB2-3HA-SGS1::kanMX4 (N17, YDG905 x YDG961)

YDG967: ho MATa cyh2r pCLB2-3HA-SGS1::kanMX4 pCLB2-3HA-MSH2::kanMX4 (N17)

YDG968: ho MATalpha cyh2r pCLB2-3HA-SGS1::kanMX4 pCLB2-3HA-MSH2::kanMX4

YDG969: ho MATa ura3 his3 leu2::NAT trp1 ade-2 can1r pCLB2-3HA-SGS1::kanMX4 pCLB23HA-MSH2::kanMX4 (W303)

YDG970: ho MATalpha ura3 his3 leu2::NAT trp1 ade-2 can1r pCLB2-3HA-SGS1::kanMX4 pCLB2-3HA-MSH2::kanMX4 (W303)

YDG979: ho/ho MATa/MATalpha ura/ura3-52 his3/his3-11 leu2::NAT/leu2-3,112 trp/trp142 ade-2/ade-2-1 can1r/can1-100 pCLB2-3HA-SGS1::kanMX4/pCLB2-3HASGS1::kanMX4 (W303, YDG866 x YDG962)

YDG980: ho/ho MATa/MATalpha cyh2r/cyh2r pCLB2-3HA-SGS1::kanMX4/pCLB2-3HASGS1::kanMX4 pCLB2-3HA-MSH2::kanMX4/pCLB2-3HA-MSH2::kanMX4 (N17, YDG967 x YDG968)

YDG981: ho/ho MATa/MATalpha ura3/ura3 his3/his3 leu2::NAT/leu2::NAT trp1/trp1 ade2/ade-2 can1r/can1r pCLB2-3HA-SGS1::kanMX4/pCLB2-3HA-SGS1::kanMX4 pCLB23HA-MSH2::kanMX4/pCLB2-3HA-MSH2::kanMX4 (W303, YDG969 x YDG970)

YDG982: ho/ho MATalpha/MATa URA3/ura3 HIS3/his3 LEU2/leu2::NAT TRP1/trp1 ADE/ade2 CAN1/can1r cyh2r/CYH2 pCLB2-3HA-SGS1::kanMX4/pCLB2-3HA-SGS1::kanMX4 pCLB2-3HA-MSH2::kanMX4/pCLB2-3HA-MSH2::kanMX4 (N17 x W303, YDG968 x YDG969) 


\section{RESOURCE AVAILABILITY}

\section{LEAD CONTACT}

Further information and requests for resources and reagents should be directed to and will be fulfilled by the Lead Contact, Jasmine Ono (j.ono@ucl.ac.uk).

\section{MATERIALS AVAILABILITY}

Strains generated in this study are available on request.

\section{DATA AND CODE AVAILABILITY}

The datasets generated during the current study have been deposited in the Dryad repository, doi:10.5061/dryad.v41ns1rtn. Source data for Figure 1A and B is provided in Extended Data Tables 1 and 2. Source data for recombination rates and Figure S1A is provided in Extended Data Table 3. Figure 1C and Figure S1B can be generated from data in Extended Data Files 1 and 2. The raw sequence data that support the findings of this study have been deposited in NCBI (SRA accession number: PRJNA672474).

\section{ACKNOWLEDGEMENTS}

We are particularly grateful to colleagues at the Max Planck Institute for Evolutionary Biology, especially Arne Nolte, Gunda Dechow-Seligmann, and Elke Bustorf, who sequenced the genomes of our hybrid strains. Mahesh Binzer-Panchal de-multiplexed the sequence reads.

Krishna B. S. Swamy provided invaluable input on data analysis. Michael Scott at University College London generously helped us with code and simulations. Finally, we thank William C. Ratcliff for his characteristically generous gesture of paying for our Dryad data deposit, saving us the trouble of arranging for a UK invoice. This project was initiated by a Royal Society International Joint Project Award to D.G. and J.-Y.L. Further support was provided by through a Max Planck Research Group Leader position to D.G., a European Molecular Biology 
Organisation Postdoctoral Fellowship (ALTF 265-2017) to J.O., and most recently by a

Leverhulme Trust Grant (RPG-2017-438) to D.G.

\section{AUTHOR CONTRIBUTIONS}

G.O.B., D.G., J.Y.-L., and J.O. conceived and directed the project. D.G. and J.Y.-L. acquired the funding. D.G. provided the laboratory resources and oversaw the project. G.O.B., J.O., J.A.D., N.H., and D.G. designed the methods employed. G.O.B., J.A.D., and D.G. coordinated the research. G.O.B. performed the data collection. G.O.B., J.O., E.K., and D.G. contributed to the statistical analyses. G.O.B., J.O., and E.K. wrote the code to perform the analyses and verified the results. G.O.B. and J.O. prepared the visualisations and performed the data curation. J.O. and D.G. wrote the original draft, G.O.B. edited the manuscript, and N.H. reviewed the manuscript.

\section{SUPPLEMENTAL REFERENCES}

S1. Grandin, N., and Reed, S.I. (1993). Differential function and expression of Saccharomyces cerevisiae B-type cyclins in mitosis and meiosis. Mol. Cell. Biol. 13, 2113-2125.

S2. Oh, S.D., Lao, J.P., Taylor, A.F., Smith, G.R., and Hunter, N. (2008). RecQ helicase, Sgs1, and XPF family endonuclease, Mus81-Mms4, resolve aberrant joint molecules during meiotic recombination. Mol. Cell 31, 324-336.

S3. Lee, B.H., and Amon, A. (2003). Role of Polo-like kinase CDC5 in programming meiosis I chromosome segregation. Science 300, 482-486.

S4. Etter, P.D., Bassham, S., Hohenlohe, P.A., Johnson, E.A., and Cresko, W.A. (2012). SNP discovery and genotyping for evolutionary genetics using RAD sequencing. In Molecular Methods for Evolutionary Genetics, V. Orgogozo and M.V. Rockman, ed. (Springer), pp. 157-178.

S5. Hohenlohe, P.A., Bassham, S., Etter, P.D., Stiffler, N., Johnson, E.A., and Cresko, W.A. (2010). Population genomics of parallel adaptation in threespine stickleback using sequenced RAD tags. PLoS Genet. 6, e1000862. 
S6. Peterson, B.K., Weber, J.N., Kay, E.H., Fisher, H.S., and Hoekstra, H.E. (2012). Double digest RADseq: an inexpensive method for de novo SNP discovery and genotyping in model and non-model species. PLoS One 7, e37135.

S7. Acinas, S.G., Sarma-Rupavtarm, R., Klepac-Ceraj, V., and Polz, M.F. (2005). PCRinduced sequence artifacts and bias: insights from comparison of two 16S rRNA clone libraries constructed from the same sample. Appl. Environ. Microbiol. 71, 8966-8969.

S8. $\quad$ Liti, G., Carter, D.M., Moses, A.M., Warringer, J., Parts, L., James, S.A., Davey, R.P., Roberts, I.N., Burt, A., and Koufopanou, V. (2009). Population genomics of domestic and wild yeasts. Nature 458, 337-341.

S9. Bergström, A., Simpson, J.T., Salinas, F., Barré, B., Parts, L., Zia, A., Nguyen Ba, A.N., Moses, A.M., Louis, E.J., and Mustonen, V. (2014). A high-definition view of functional genetic variation from natural yeast genomes. Mol. Biol. Evol. 31, 872-888.

S10. Li, H. (2013). Aligning sequence reads, clone sequences and assembly contigs with BWA-MEM. arXiv, arXiv:1303.3997.

S11. Anderson, C.M., Chen, S.Y., Dimon, M.T., Oke, A., DeRisi, J.L., and Fung, J.C. (2011). ReCombine: a suite of programs for detection and analysis of meiotic recombination in whole-genome datasets. PloS One 6, e25509.

S12. RCore, T.E.A.M. (2016). R: A language and environment for statistical computing. R Foundation for Statistical Computing. Vienna, Austria.

S13. Virtanen, P., Gommers, R., Oliphant, T.E., Haberland, M., Reddy, T., Cournapeau, D., Burovski, E., Peterson, P., Weckesser, W., Bright, J., et al. (2020). SciPy 1.0: fundamental algorithms for scientific computing in Python. Nat. Methods 17, 261-272.

S14. Cherry J.M., Hong E.L., Amundsen C., Balakrishnan R., Binkley G., Chan E.T., Christie K.R., Costanzo M.C., Dwight S.S., Engel S.R., et al. (2012). Saccharomyces Genome Database: the genomics resource of budding yeast. Nucleic Acids Res. 40, D700-D705.

S15. Kao, K.C., Schwartz, K., and Sherlock, G. (2010). A genome-wide analysis reveals no nuclear Dobzhansky-Muller pairs of determinants of speciation between $S$. cerevisiae and S. paradoxus, but suggests more complex incompatibilities. PLoS Genet. 6, e1001038. 